Indian J. Anim. HIth. (2018), 57(1) : 103-108

Short Communication

\title{
AN OUTBREAK OF INFECTIOUS CAPRINE KERATO-CONJUNCTIVITIS IN A FLOCK OF GOATS - CASE REPORT
}

\author{
G. S. PANDEY \\ Department of Disease Control \\ School of Veterinary Medicine, University of Zambia \\ P.O. Box 32379, Lusaka, Zambia
}

Infectious caprine kerato-conjunctivitis (ICK) is a highly contagious and multifactorial bacterial disease of goats having enormous difference in the clinico-pathological picture. A total of six crossbred young Boer goats out of 124 in a flock, showing emaciation, corneal opacity and blindness at a farm in Lusaka district of Zambia, when clinically examined. Clinical examination revealed mucopurulent ocular discharge, corneal opacity and partial to complete blindness. Corneal swabs culture yielded Mycoplasma spp. and Pseudomonas aureginosa from all six goats. The diagnosis of ICK arrived on the basis of clinical signs and laboratory investigation. The treatment consisting of daily eyewash with clean warm water, a single injection of long-acting oxytetracycline and application of oxytetracycline opthalmic ointment, three times a day resulted in the recovery and vision restoration of all six goats within 8-12 days. This warrants farmers and veterinarians to practice prevention and treatment when noticed among goats. To the best knowledge of the author, this is first documented report of infectious caprine keratoconjunctivitis (ICK) among goats in Zambia.

Key words: Goats, Infectious kerato-conjunctivitis, Mycoplasma spp., Oxytetracycline ointment, Zambia

Infectious caprine kerato-conjunctivitis (ICK) is a highly contagious and multifactorial bacterial disease of goats having enormous difference in epidemiological and clinicopathological picture (Shahzad et al., 2013). It has been well documented that ICK is influenced by several factors including immune status of 
the predisposed animal, virulence of the pathogenic organisms involved, environmental factors and secondary infections (Abdullah et al., 2015; Fernandez-Aguilar et al., 2017). From Africa, the disease has been reported among goats in Nigeria, Kenya, Tanzania, Zimbabwe and South Africa (Osuagwuh and Akpokodie, 1979; Sibande et al., 1992; Shija et al., 2014). Inflammation of cornea, conjunctiva and finally ulceration are the main features of this disease and may cause temporary to permanent blindness in infected animals (Abdullah et al., 2013). Among sheep and goats, the etiological agents of this disease incriminated are Mycoplasma spp., Chlamydophila spp., Staphylococcus aureus, Moraxella caprae, Moraxella ovis, Listeria monocytogenes, Pseudomonas aeruginosa (Kodjo et al., 1995; Laven and Lawrence, 2006; Abdullah et al., 2014; Abdullah et al., 2015; Jesse et al., 2017). In cattle, the most important causative agent of infectious bovine keratoconjuctivitis is Moraxella bovis (Frisch, 1975), but this pathogen does not affect goats. The reported clinical signs associated with ICK are mainly mucopurulent ocular discharge, blepharospasm, swollen eyes, corneal opacity, photophobia (Browning, 2007). Review of the published data has indicated that no study has been reported in Zambia on ICK among goats. In the present communication, an outbreak of ICK among goats in Zambia with successful treatment regime is being reported.

During the month of February 2017 in Lusaka district of Zambia, among a flock of 124 goats, a total of six young Boer crossbred goats were reported to have anorexia, weakness, lacrimation and unable to see and graze for the last 10 days. These affected goats ranged from six months to one year and weighed $12 \mathrm{Kg}-18 \mathrm{Kg}$. The author visited the farm on the request of farmer and did the detailed clinical examination of the affected goats. The goats were shifted from another farm to this farm a month ago.

Corneal swabs were collected from the affected eyes using the sterile aseptic procedure for microbiological examination. The blood was also collected from each affected goat in EDTA tube from jugular vein for hematological evaluation. The swabs were inoculated on primary bacteriological culture and based on colony the selective media were used. The standard mycoplasma broth medium enriched with $20 \%$ horse serum, $2.5 \%$ yeast extract and $1 \%$ glucose used for mycoplasma isolation. The resulting growth was propagated at $37^{\circ} \mathrm{C}$ in a humified atmosphere having $5 \%$ carbon dioxide. For pseudomonas isolation peptone agar was used containing 5\% defibrinated sheep blood. Hematological parameters were evaluated using routine hematology procedure. 
Indian Journal of Animal Health, June, 2018

Infectious caprine kerato-conjunctivitis in goats

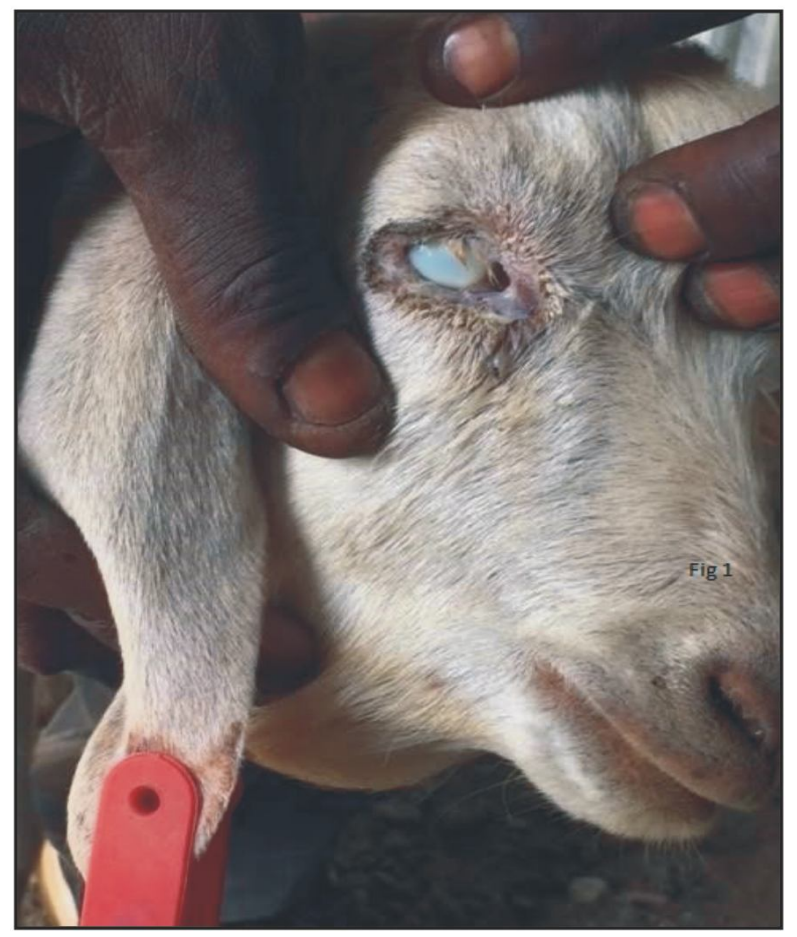

Fig. 1. Goat image showing marked corneal opacity and loss of vision

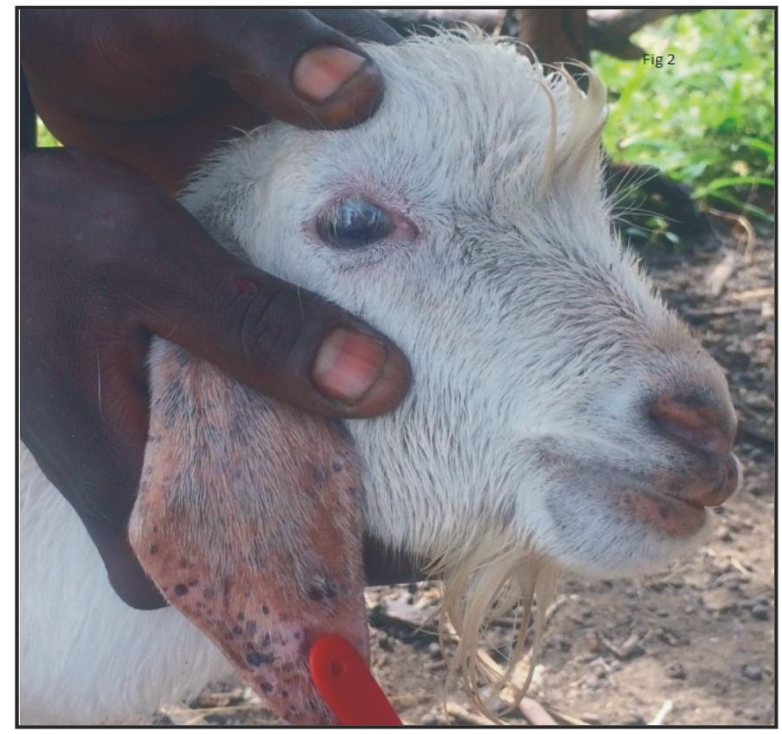

Fig. 2. Goat image showing disappearance of opacity of cornea after treatment and restoration of vision 
Affected goats then were separated and kept in isolation. Feed and water was provided since they were not able to graze. Eyes of all six goats were cleaned with clean warm water daily three times a day and thereafter applied oxytetracycline opthalmic ointment with polymyxin B sulphate (terramycin ophthalmic ointment) three times a day for continuous eight days. One dose of longacting oxytetracycline (Oxy 20\% LA) @ $20 \mathrm{mg}$ per $\mathrm{kg}$ body weight was given $\mathrm{i} / \mathrm{m}$ to each affected goat on the initial day of treatment.

The clinical examination had revealed, emaciation, weakness, rough coat, the slightly elevated temperature in 4 of them (40.0- $41.0^{\circ} \mathrm{C}$ ). In three of them, both eyes were affected while in other three only one eye was affected. A thorough examination of eyes in all six goats revealed mucopurulent discharge, yellow-green pus turning into the crust around eyes, redness and complete corneal opacity of both eyes in three goats (Fig. 1) and partial opacity and blindness in three goats. There was absence of pupillary reflex response or blinking of eyes when a finger brought nearer to the affected eyes, indicating loss of vision. A tentative diagnosis of keratoconjunctivitis was made based on clinical signs. Hematological examination revealed leukocytosis in four goats. Pure culture of Mycoplasma spp. and Pseudomonas aeruginosa was isolated from all six cases.Treatment as indicated resulted in recovery of all cases within 8-12 days of the treatment showing with restoration of vision and disappearance of mucopurulent discharge and corneal opacity (Fig. 2).

Livestock farming is an important economical and subsistence farming in Zambia. Goats are kept by poorer strata of the population in rural areas. Most of the rural people are directly or indirectly associated with goat farming and use as a bank in need of funds for emergency needs. Infectious kerato-conjunctivitis is serious threat to the small ruminants farming throughout the world (Abdullah et al., 2013). This disease although present among cattle in Zambia (Annual Report, 2015), has not been reported among goats. Moreover Moraxella bovis the etiological agent of bovine kerato-conjunctivitis does not affect goats. The eyes become cloudy due to oedema and infiltration of leucocytes and with passage of time lead to the prolapse of iris and corneal ulcer that results to the loss of vision (Abdullah et al., 2014). The clinical signs observed in this outbreak are consistent with earlier studies (Abdullah et al., 2013; Mughal et al., 2015; Jesse et al., 2017). This disease manifests throughout the seasons of year, however in the light of some published data, it is more commonly seen in winter due to housing and close contact while feeding and mating (Aitken, 2007; Scott, 2010), however in the current outbreak the disease occurred in rainy season at a new farm with recent shifting of goats from another farm. Transportation 
being one of the causes of stress might have precipitated the disease (Abdullah et al., 2014). As the disease progresses without treatment, ocular discomfort and visual disturbance may lead to inappetance or inability to locate food and these will then consequently affect the body condition score resulting in emaciation (Jesse et al., 2017) as was observed in the present outbreak. Both systemic and topical antibiotic treatments have been administered in the present case as previously recommended (Whittier et al., 2009; Jesse et al., 2017). The use of systemic and topical antibiotics helps the recovery (Abdullah et al., 2014; Jesse et al., 2017) as was the case in the present study. To the best knowledge of the author, this is first documented report of infectious caprine kerato-conjunctivitis among goats in

\section{REFERENCES}

Abdullah FFJ, Naidu NRG, Sadiq Y, Abba $\mathrm{Y}$ and Tijjani A et al., 2015. Prevalence of Moraxella ovis infection in goats. IOSR J Agri Vet Sci, 8(11): 99-102

Abdullah FFJ, Radzuan NS, Tijjani, A, Adamu, L and Abba, Y et al., 2014.
Zambia. This warrants farmers and veterinarians to practice prevention and treatment when noticed without delay to avoid spread, production losses and mortality. Incidence of kerato-conjunctivitis and the microorganisms involved are linked to predisposing and environmental factors which are important risk factors to consider when correct treatment and preventive measures are determined. The chances for recovery are high if cases of ICK are reported in time and immediate treatment instituted as reported in the present case.

\section{ACKNOWLEDGMENT}

The author is grateful to the laboratory staff for technical assistance and the farmer for supporting the investigation and following the advice on treatment.

Stage II kerato-conjunctivitis in a goat: A case report. IOSR J Agric Vet Sci, 7(1): 16-18

Abdullah FFJ, Adamu L, Osman AY and Saharee AA, 2013. Clinical management of stage II infectious kerato-conjunctivitis associated with 
Staphylococcus aureus in a dairy cow. IOSR J Agric Vet Sci, 4: 69-73

Annual Report, 2015. Department of Veterinary Services, Lusaka, Zambia

Aitken OID, 2007 Diseases of sheep. Oxford, UK, Wiley-Blackwell, pp 342-345

Browning ML, 2007. Kerato-conjunctivitis (pink eye) in goats. Extension Animal Scientist, Alabama A\&M University, 3: $88-90$

Fernandez-Aguilar X, Rossi L, Cabezon O, Giorgino A and Llopis IV et al., 2017. Infectious keratoconjunctivitis and occurrence of Mycoplasma conjunctivae and Chlamydiaceae in small domestic ruminants from Central Karakoram, Pakistan. Vet Record, 181:237

Frisch JE, 1975. The relative incidence and effect of bovine infectious keratoconjunctivitis in Bos indicus and Bos taurus cattle. Anim Prod, 21: 265274

Jesse FFA, Chung ELT, Abba Y, Bitrus AA, Hambali IU et al., 2017. Clinical management of stage I pinkeye with concurrent pneumonic pasteurellosis in a goat: A case report. J Adv Vet Anim Res, 4(4): 390-393

Kodjo A, Tonjum T, Richard Y and Bovre K, 1995. Moraxella caprae a new member of the classical Moraxellae spp. with very close affinity to Moraxella bovis. J Sys Bact, 45(3): 467-471
Laven RA and Lawrence KR, 2006. An outbreak of iritis and uveitis in dairy cattle at pasture associated with the supplementary feeding of baleage. New Zealand Vet J, 54(3): 151-152

Mughal MN, Anwar A, Abbas G and Azeem W, 2015. Infectious caprine keratoconjunctivitis in a Beetal buck: A Case report. Int J Mol Vet Res, 5:1-3

Osuagwuh AI and Akpokodie JU, 1979. Infectious kerato-conjunctivitis in goats and sheep in Nigeria. Vet Rec, 105(6): $125-126$

Scott PR, 2010. Cattle and Sheep Medicine. Manson Publishing Ltd., London, UK, pp 265-267

Shahzad W, Munir R, Rana MY, Ahmad R and Khan MS et al., 2013. Prevalence, molecular diagnosis and treatment of Mycoplasma conjunctivae isolated from infectious kerato-conjunctivitis affected Lohi sheep maintained at Livestock Experiment Station, Bahadurnagar, Okara, Pakistan. Trop Anim Hlth Prod, 45(3): 737-742

Shija DSN, Kusiluka LJM, Chenyambaga SW, Shayo D and Lekule FP, 2014. Animal health constraints in dairy goats kept under small holder dairy system in Kongwa and Mvomera districts, Tanzania J Vet Med Anim Hlth, 6(11): 268-279

Sibande LM, Ndlovu LR and Bryant MJ, 1992. Some experience in adapting previously free ranging traditionally 
managed Matabele goats of Zimbabwe to individually stall feeding. Proceedings of African Small Ruminant Res Network, Nairobi, Kenya, 10-14 ${ }^{\text {th }}$ December 1992. pp 177-184
Whittier WD, Currin N and Currin J, 2009. Pinkeye in beef cattle, Virginia Cooperative Extension, College of Agriculture and Life Sciences, Virginia Polytechnic Institute and State University, publication 400-750 